Psychotherapeut 2010 · 55:189-193

DOI 10.1007/s00278-010-0747-6

Online publiziert: 30 . April 2010

(c) Springer-Verlag 2010
Thomas Fydrich ${ }^{1}$ Alexandra Martin ${ }^{2}$

${ }^{1}$ Institut für Psychologie, Humboldt-Universität zu Berlin,

2 Psychosomatik: Psychotherapieforschung, Universitätsklinikum

Erlangen, Universität Erlangen-Nürnberg, Erlangen

\title{
Schwerpunktheft zum Thema Somatopsychologie
}

Somatopsychologie? Schon wieder ein neues Fach der Psychologie? Und wieso nicht Psychosomatik? Die gibt es doch schon lange und ist als Begriff und als Modell der Erklärung und Behandlung von körperlichen Störungen unter Berücksichtigung psychischer Faktoren schon etabliert. Außerdem haben wir doch die Gesundheitspsychologie und auch die Verhaltensmedizin oder auch die Rehabilitationspsychologie als Forschungs- und Anwendungsbereiche, die sich mit körperlicher Gesundheit, Prävention, psychischen Faktoren der Entstehung und des Verlaufs von somatischen Erkrankungen und eben mit der Rehabilitation beschäftigen. Warum also ein neues Fach?

Im Frühjahrsrennen 2010 der GoogleTreffer schaffen es allein in der deutschsprachigen Liga die Psychosomatik auf über 600.000, die Gesundheitspsychologie auf knapp 100.000 und die Verhaltensmedizin auf etwa 50.000 Einträge. Weit abgeschlagen folgt die Somatopsychologie mit gerade mal 4000 Hits. Wir haben uns entschlossen, mit einem Schwerpunktheft zur „Somatopsychologie“ diese Relation zu ihren Gunsten zu verbessern. Ziel ist dabei, im Vergleich zu den schon etablierten Fächern und Begriffen auf wichtige Unterscheidungen hinzuweisen und den Fokus in besonderer Weise auf die Psychologie und Psychotherapie als Disziplinen zu richten, die sowohl gut begründete theoretische Modelle, empirische Befunde als auch gut evaluierte psychotherapeutische Interventionen im Bereich der Behandlung (primär) somatischer Erkrankungen entwickelt haben. Es besteht nach wie vor umfassender For- schungsbedarf über das Zusammenspiel psychischer und somatischer Faktoren bei der Entstehung, dem Verlauf und der Behandlung körperlicher Erkrankungen. Interessant ist, dass die bekannten Befunde über solche Zusammenhänge teilweise schon vor langer Zeit erhoben wurden, dass aber solche Kenntnisse in der konkreten Gesundheitsversorgung chronisch körperlich Kranker praktisch kaum umgesetzt werden. Vor allem betrifft dies die ambulante Versorgung. In diesem $\mathrm{Zu}$ sammenhang wird von den Psychotherapeutenkammern der Länder und von der Bundespsychotherapeutenkammer seit einiger Zeit die Einführung der „klinischen Somatopsychologie" als curriculares Fortbzw. Weiterbildungsfach diskutiert.

\section{Somatopsychologie: Definition}

Gegenstand der Somatopsychologie ist die Grundlagen- und Interventionsforschung zu psychologischen und sozialen Faktoren, die bei Entstehung, Verlauf, Behandlung, Prävention und Rehabilitation primär somatischer Erkrankungen eine Rolle spielen. Die auf dieser Forschung beruhende Entwicklung und Evaluation psychologischer bzw. psychotherapeutischer Interventionen und Präventionsmaßnahmen wird als klinische Somatopsychologie bezeichnet.

\section{Unterschiede zu anderen Disziplinen und Fächern}

Was unterscheidet nun die Somatopsychologie von schon bekannten, v. a. von folgenden Fächern:
- psychosomatische Medizin,

- Verhaltensmedizin und

- Gesundheitspsychologie?

\section{Psychosomatik und psychosomatische Medizin}

Der Begriff der Psychosomatik lässt sich historisch v. a. auf die Psychoanalyse zurückführen. Georg Groddeck (1923) verstand in seinem Buch vom Es körperliche Symptome von Krankheiten als Symbole, mit denen sich das „Es“ als Triebkraft, die durch die Lebensumstände, die Gesellschaft und die Moral unterdrückt wird, Ausdruck verschafft. Vergleichbar hatten schon Freud u. Breuer (1895) in ihren Studien über Hysterie Konversionssymptome (v. a. dort die körperlichen Symptome) als Zeichen psychischer Erregung und Konflikte verstanden, die nicht angemessen verarbeitet, abgeführt bzw. gelöst werden konnten.

Als der eigentliche „Vater“ der psychosomatischen Medizin kann jedoch der in Ungarn 1891 geborene und 1964 in Kalifornien gestorbene Franz Alexander gelten. Er lebte von 1919 bis 1930 in Berlin und war dort der erste Ausbildungskandidat des Berliner Psychoanalytischen Instituts. Im Jahr 1950 erfolgte die Erstveröffentlichung des „Klassikers“ mit dem Titel Psychosomatic Medicine, in der er die aus heutiger Sicht klassischen „Psychosomatosen" beschrieb (Alexander 1950). Zu diesen „holy seven“" (klassischen Sieben) gehören:

- Colitis ulcerosa,

- Ulcus pepticum,

- Asthma bronchiale, 
- essenzielle Hypertonie,

- atopische Neurodermitis,

- Hyperthyreose und

- rheumatoide Arthritis.

Die klassische psychosomatische Medizin hat ihre Wurzeln eindeutig in der Psychoanalyse. Auf der Grundlage von Triebtheorien und Charaktermodellen wird implizit und explizit die Gültigkeit der Neurosenlehre und dort v. a. des Konversionsmodells angenommen: Somatische Symptome oder Erkrankungen lassen sich danach auf psychische innere Konflikte (meist zwischen den von Freud erstmals angenommenen Instanzen des Ich, Es und Über-Ich) oder Konflikte mit der (sozialen) Umwelt zurückführen. Konflikte werden danach in symbolischer Form durch körperliche Symptome ausgedrückt, wobei diese Ausdrucksform dem Patienten unbewusst seien. Zudem wurde (nach Alexander) von einer Spezifität der „Organsprache“ ausgegangen: Jedem Körpersymptom wurde eine besondere Konfliktsituation zugeordnet.

Historisch für die psychosomatische Medizin bedeutsame Daten waren die Gründung der American Psychosomatic Society (1942) und etwas später der Start des Journal of Psychosomatic Medicine. In Deutschland war die „Heidelberger Psychosomatik“ mit der ersten stationär-universitären Psychosomatik (von Weizäcker, Mitscherlich) bedeutsam; die Besetzung des ersten Lehrstuhls für psychosomatische Medizin erfolgte im Jahr 1962 mit Horst-Eberhard Richter in Gießen. Das Lehrbuch der psychosomatischen Medizin von Thure von Uexküll (1979 in der Erstauflage) entwickelte sich zum Standardwerk des Lehrfaches.

In der weiteren Entwicklung des Fachs „psychosomatische Medizin“ wurden die, auf der Neurosenlehre basierenden Positionen teilweise relativiert - z. B. durch das offenere „biopsychosoziale“ Modell von Georg W. Engel (1977) und um soziologische und philosophische Aspekte erweitert. Aktuell spielen auch systemische Ansätze in den theoretischen Konzeptualisierungen eine Rolle. Dennoch nehmen aktuelle Definitionen der „psychosomatischen Medizin“ als wesentliches Moment psychische und psychosoziale Faktoren als Ursache von somatischen Krankheiten mit auf. So heißt es im Text der Musterweiterbildungsordnung der Bundesärztekammer: „Das Gebiet Psychosomatische Medizin und Psychotherapie umfasst die Erkennung, psychotherapeutische Behandlung, Prävention und Rehabilitation von Krankheiten und Leidenszuständen, an deren Verursachung psychosoziale und psychosomatische Faktoren einschließlich dadurch bedingter körperlichseelischer Wechselwirkungen maßgeblich beteiligt sind" (Musterweiterbildungsordnung der Bundesärztekammer 2003 i.d.F. von 2008, S. 120).

Damit ist nach wie vor das klassische ätiologieorientierte Modell, das weitgehend von einer Annahme der psychogenen Verursachung vieler („psychosomatischer") körperlichen Erkrankungen ausgeht, bestimmend für den Bereich und das Fach der psychosomatischen Medizin.

\section{Verhaltensmedizin}

Im ersten Kapitel ihres gleichnamigen Lehrbuchs definiert Ehlert (2003, S. 4) Verhaltensmedizin als „die klinische Anwendung von Techniken, die aus der experimentellen Analyse von Verhalten abgeleitet und zur Evaluation, Prävention und Behandlung körperlicher Erkrankungen oder physiologischer Funktionsstörungen eingesetzt werden. Zum Gegenstandbereich der Verhaltensmedizin gehört im Weiteren die empirische Erforschung der Zusammenhänge zwischen Verhalten, somatischen Erkrankungen und Problemen der Gesundheitsversorgung“. Die wesentlichen Merkmale der Verhaltensmedizin liegen in der Interaktion zwischen psychischen Vorgängen und körperlichen Prozessen sowie in der interdisziplinären, empirischen Auseinandersetzung mit Gesundheit und Krankheit.

Verhaltensmedizin ist ein interdisziplinäres Feld, das sich mit der Entwicklung und Integration von Wissen und Interventionstechniken v. a. aus den Verhaltens- und biomedizinischen Wissenschaften beschäftigt. Es trägt zum Verständnis der Entstehung, des Verlaufs sowie der Behandlung von Krankheiten sowie zum Erhalt von Gesundheit bei. Im Rahmen der Verhaltensmedizin werden interdisziplinäre Interventionsansätze zur
Prävention, Diagnostik, Behandlung und Rehabilitation von Erkrankungen entwickelt (Ehlert 2003; Definition der Society of Behavioral Medicine). Wissenschaftstheoretisch und forschungsmethodologisch werden in der Verhaltensmedizin empirische und v. a. experimentelle Forschungsmethoden ebenso betont wie der Einsatz verhaltenstherapeutischer Interventionsmodelle und -methoden.

Der Begriff „behavioral medicine“ wurde erstmals zu Beginn der 7oer Jahre von Lee Birk (1973) im Titel einer Publikation genutzt: „Biofeedback: behavioral medicine“. Die multikausale Sichtweise hinsichtlich der Entstehung und des Verlaufs von Erkrankungen sowie der Erhaltung von Gesundheit war ein wichtiges Anliegen einer für die Verhaltensmedizin wegweisenden Konferenz der Yale University in den USA im Jahr 1977. Auf diese Konferenz geht die erste Definition und nähere Gegenstandsbestimmung von „behavioral medicine" zurück:

„Behavioral medicine is the field concerned with the development of behavioral-science knowledge and techniques relevant to the understanding of physical health and illness and the application of this knowledge and these techniques to diagnosis, prevention, treatment and rehabilitation. Psychosis, neurosis and substance abuse are included only insofar as they contribute to physical disorders as an end point" (Schwartz u. Weiss 1978).

Historisch ist die Verhaltensmedizin deutlich jünger als die Psychosomatik. Die Gründung der „Society of Behavioral Medicine" geht zurück auf die oben genannte Yale-Konferenz am Ende der siebziger Jahre. Ebenfalls in dieser Zeit startete das Journal of Behavioral Medicine als Fachorgan der Gesellschaft. Die „Deutsche Gesellschaft für Verhaltensmedizin und Verhaltensmodifikation“ wurde 1984 gegründet; namhafte Personen in der Gründungsphase waren Nils Birbaumer, Wolfgang Miltner und Irmela Florin. Das erste Lehrbuch zur Verhaltensmedizin wurde 1986 von Miltner, Birbaumer und Gerber herausgegeben. International schlossen sich 1992 nationale Verbände der Verhaltensmedizin zur „International Society of Behavioral Medicine (ISBM)“ zusammen, die zweijährlich internationale Kongresse durchführt; zwei davon schon in 
Deutschland (1992 in Hamburg und 2004 in Mainz).

\section{Gesundheitspsychologie}

Die Gesundheitspsychologie versteht sich als Teilgebiet der Psychologie. Zentrale Fokusse sind die Entwicklung und empirische Überprüfung von theoretischen Modellen zur Erhaltung von Gesundheit. Dabei werden in besonderem Maß Anleihen an theoretische Modelle der Sozialpsychologie gemacht. Es geht um die Erforschung von personbezogenen, sozialen und strukturellen Einflussfaktoren auf die körperliche und psychische Gesundheit. Zentrale Themen der Gesundheitspsychologie sind die Frage nach den Determinanten für gesundheitsförderliches bzw. Gesundheit gefährdendes Verhalten und damit verbunden die Entwicklung und Evaluation von psychologischen Interventions- bzw. Präventionsmaßnahmen zum Erhalt und zur Förderung der Gesundheit.

Schwarzer (2004) definiert Gesundheitspsychologie als Beitrag der Psychologie zur:

- Förderung und Erhaltung von Gesundheit,

- Verhütung und Behandlung von Krankheiten,

- Bestimmung von Risikoverhaltensweisen,

- Diagnose und Ursachenbestimmung von gesundheitlichen Störungen,

- Rehabilitation und

- Verbesserung des Systems gesundheitlicher Versorgung.

Auch die Gesundheitspsychologie ist eher jung. Sie versteht sich weniger als interdisziplinäres Fach (wie die Verhaltensmedizin), sondern gründet sich auf die Anwendung psychologischer Modelle sowie Forschungs- und Interventionsmethoden zum Thema Gesundheit. Historisch kann die Gesundheitspsychologie auf die Gründung der „Division of Health Psychology" in der American Psychological Association im Jahr 1978 zurückgeführt werden. In den USA gehört die Gesundheitspsychologie längst zu den Standarddisziplinen in der Psychologie. Die Gründung der European Health Psychology Society (EHPS) erfolgte 1986; eben- falls richtete die International Association for Applied Psychology (IAAP) 1986 ihre Fachgruppe Health Psychology ein. Als Seniorperiodikum des Faches erscheint seit 1982 die Zeitschrift Health Psychology, der Start einer ganzen Reihe internationaler Zeitschriften folgte (u. a. Psychology and Health, International Journal of Health Psychology). In Deutschland wurde 1992 die Fachgruppe Gesundheitspsychologie in der Deutschen Gesellschaft für Psychologie (DGPs) gegründet. Namhafte Personen „der ersten Stunde" in Deutschland sind Ralf Schwarzer (Freie Universität Berlin), Walter Krohne (Mainz) und Peter Schwenkmezger (Trier). Gleich nach der ersten Fachgruppentagung in Berlin folgte auch die erste Ausgabe der Zeitschrift für Gesundheitspsychologie im Jahr 1993.

\section{Zusammenfassender Vergleich und Plädoyer für das Fach Somatopsychologie sowie eine curriculare Fort- bzw. Weiterbildung „klinische Somatopsychologie"}

Alle vier dargestellten Disziplinen (Psychosomatik, Verhaltensmedizin, Gesundheitspsychologie und Somatopsychologie) gehen von der Prämisse aus, dass psychischen Faktoren eine zentrale Rolle bei der Entstehung und Bewältigung von Krankheiten zukommt.

Die Psychosomatik wird v. a. durch die psychosomatische Medizin vertreten. Sie ist historisch ein Forschungs- und Anwendungsgebiet, das auf psychodynamische Ätiologiemodelle für somatische Erkrankungen zurückgeht. Nach wie vor spielen ätiologieorientierte Fragestellungen, bei denen psychische Faktoren ursächlich für die Entstehung und den Verlauf körperlicher Erkrankungen gesehen werden, eine zentrale Rolle. Psychosomatik ist weitgehend ein Fach der Medizin.

Die Verhaltensmedizin ist ein interdisziplinäres Fach, das v. a. theoretische Modelle, Methoden und Erkenntnisse aus Psychologie und Medizin integriert. Mitglieder entsprechender Fachverbände sind daher zu gleichen Anteilen v. a. Mediziner und Psychologen. Hinsichtlich der aus den Modellen und Forschungsbefunden abgeleiteten Behandlungsansät- ze überwiegen solche, die der klinischen Verhaltenstherapie verpflichtet sind.

Die Gesundheitspsychologie ist dagegen nicht interdisziplinär. Sie versteht sich als psychologisches Fach, das primär den Erhalt und das Wiedererlangen von Gesundheit zum Gegenstand hat. Dies geschieht unter Rückgriff auf andere psychologische Disziplinen, v. a. der Sozialpsychologie.

Auch die Somatopsychologie ist ein primär psychologisches Fach. Gegenstand ist die Erforschung von psychischen Faktoren, die bei der Entstehung und dem Verlauf von somatischen, v. a. chronisch körperlichen Erkrankungen eine Rolle spielen. Als klinische Somatopsychologie sind ihre Themen zudem die Entwicklung und Überprüfung von psychotherapeutischen Behandlungsansätzen bei körperlichen Erkrankungen, die Verbesserung der Versorgung sowie die Aus-, Fort- und Weiterbildung in diesem Bereich.

Obwohl Forschungsergebnisse zeigen, dass sich psychologische (v. a. Verhaltensfaktoren) im Zusammenhang mit körperlichen Erkrankungen durch systematische psychologische Interventionen günstig beeinflussen lassen, bekommen die meisten Patienten mit somatischen Erkrankungen, bei denen psychologische Faktoren - v. a. auf den Verlauf einer Erkrankung - eine Rolle spielen, im regulären Versorgungssystem keine psychologische bzw. psychotherapeutische Begleitbehandlung. Zur Verbesserung dieser Situation müssen auch die fachlichen Voraussetzungen bei psychologischen und ärztlichen Psychotherapeuten bzw. bei Kinder- und Jugendlichenpsychotherapeuten gewährleistet sein. Aktuell sind die Inhalte und die praxisorientierte Ausbildung nicht in ausreichendem $M a ß$ in die Psychotherapieausbildungen integriert. Daher wird eine curriculare Fortbildung oder eine Weiterbildung in klinischer Somatopsychologie für diese Berufsgruppen als sinnvoll erachtet.

Zum Tätigkeitsfeld einer etablierten klinischen Somatopsychologie (Bundespsychotherapeutenkammer 2006) gehören insbesondere:

a. Die diagnostische Beurteilung der kognitiven, emotionalen, behavioralen und sozialen Faktoren, die Einfluss auf die somatische Krankheit und ihre Be- 
wältigung nehmen. Dabei sind biomedizinische Faktoren der Genese und Aufrechterhaltung der Erkrankung zu berücksichtigen.

b. Die Planung, Durchführung und Evaluation geeigneter psychotherapeutischer Behandlungen, einschließlich der Unterstützung bei der Krankheitsverarbeitung. Hierzu gehört auch die beratende oder ggf. therapeutische Einbeziehung des sozialen Umfelds.

c. Die Unterstützung psychosozialer Rehabilitation.

d. Die Erstellung von Gutachten.

Von verschiedenen Fachverbänden und einzelnen Psychotherapeutenkammern wurde für den Bereich der psychotherapeutischen Behandlung von Patienten mit v. a. chronischen und schweren körperlichen Erkrankungen eine Reihe spezifischer Curricula bzw. Weiterbildungsordnungen für Fort- und Weiterbildungen entwickelt. Beispiele sind die psychologische Schmerztherapie, Psychoonkologie, Pschodiabetologie, Psychokardiologie, Psychodermatologie, Psychogynäkologie, Biofeedback-Therapie, Rehabilitationspsychologie oder das Curriculum Verhaltensmedizin.

Bestandteile einer umfassenden curricularen Fortbildung für Psychotherapeuten sollten sein:

1. Allgemeine Grundlagen der Somatopsychologie (theoretische Modelle, Forschungsmethoden, Epidemiologie, Interventionsmethoden, Chronifizierung somatischer Erkrankungen, allgemeine und spezifische psychologische Diagnostik und Differenzialdiagnostik, psychologisch-psychotherapeutische Interventionen zur Förderung des Gesundheitsverhaltens und der Krankheitsbewältigung, Psychoedukation, Krisenintervention, Kurzzeittherapie, Gruppeninterventionen, rechtliche Grundlagen und sozialmedizinische Begutachtung, medizinische und psychosoziale Versorgungssysteme, interdisziplinäre Kooperation, Evaluation und Qualitätsmanagement).

2. Biomedizinische Grundlagen, medizinische Diagnostik und Intervention für die jeweilige somatische Erkrankung.

3. Spezielle Anwendungsbereiche der klinischen Somatopsychologie mit der Möglichkeit der Schwerpunktbildung (z. B. Schmerztherapie, Psychokardiolo- gie, Psychodiabetologie, Psychoonkologie, Psychodermatologie).

4. Klinische Tätigkeit und Supervision in Institutionen der medizinischen und/ oder psychosomatischen Versorgung, in denen schwerpunktmäßig Patienten mit somatischen Problemen aus den genannten Tätigkeitsfeldern behandelt werden; Erstellung von Gutachten.

\section{Zu diesem Sonderheft}

Das vorliegende Sonderheft zur Somatopsychologie gibt exemplarisch Einblick in einige Forschungs- und Interventionsbereiche.

Den Auftakt bildet eine Übersichtsarbeit von Vögele zu psychischen Störungen, Diagnostik und Intervention beim ,acquired immune deficiency syndrome" (Aids) und Infektionen mit dem „human immunodeficiency virus“(HIV). Nach Einführung in Krankheitsbild und medizinische Behandlung wird das Problem der erhöhten psychischen Belastung von HIV-infizierten Personen aufgegriffen, und klinische Anwendungsbereiche werden illustriert: Dazu gehören Interventionen zur Verbesserung der Behandlungsadhärenz, zum Abbau von Risikoverhalten und zur Gesundheitsförderung (z. B. Stressmanagement) genauso wie die Psychotherapie bei komorbiden psychischen Störungen.

Die Übersichtsarbeit zu gastrointestinalen Erkrankungen, insbesondere zum Reizdarmsyndrom (Hefner et al.) soll die Breite der auf das Krankheitsgeschehen und -erleben Einfluss nehmenden psychologischen Faktoren verdeutlichen. Dazu gehören die Interaktionen von Stress bzw. negativer Emotionen und somatischer Prozesse genauso wie Placebowirkung, Lerneffekte und schließlich psychotherapeutische Ansätze und ihre Evidenzbasierung.

Bei vielen chronischen körperlichen Krankheiten wird inzwischen betont, dass subjektive Krankheitskonzepte der Betroffenen die erlebte Belastung und teilweise auch den Verlauf der Erkrankungen beeinflussen und von daher potenziell therapierelevant sind. In der Originalie von Krille et al. wird eine Studie zu dem Zusammenhang von subjektiven Krankheitskonzepten und Krankheitsbelastung bei chronisch-entzündlichen Darmerkrankungen (CED) vorgestellt.

Die Biofeedback-Behandlung kommt inzwischen bei verschiedenen somatischen Beschwerdebildern, insbesondere chronischen Schmerzen, zum Einsatz verbunden mit dem Ziel, die Symptomatik über die Selbstkontrolle körperlicher Prozesse (sowie verbesserte Selbstkontrollerwartungen der Betroffenen) positiv zu beeinflussen. Vorgestellt werden hier ein aus Biofeedback und kognitiver Verhaltenstherapie kombiniertes Vorgehen bei kraniomandibulären Schmerzen und Dysfunktionen (CMD) sowie erste Ergebnisse der Wirksamkeit im Vergleich zu zahnmedizinischer Standardbehandlung (Shedden Mora et al.).

Eine weitere Originalie befasst sich mit der psychischen Belastung von Tinnituspatienten (Schaaf et al.) und leitet ein gestuftes therapeutisches Vorgehen ab. Neben Indikationen und Interventionen verschiedener psychotherapeutischer Ansätze stellen die Autoren die Evaluation ihrer Kombination aus tiefenpsychologisch und verhaltenstherapeutisch orientierter Behandlung bei Tinnitus dar.

Die Psychotherapie bei chronischen vulvovaginalen Schmerzen, einem bislang wenig bekannten Krankheitsbild, wird anhand eines Fallbeispiels (Härtl et al.) in der Rubrik Behandlungsprobleme illustriert. Auch wenn die Ätiologie der Vulvodynie bislang nur in Ansätzen bekannt ist, zeigt der Fall, dass sich im Rahmen der hier beschriebenen Verhaltenstherapie sowohl die Bewältigungsstrategien im Umgang mit den Schmerzen und dem Juckreiz als auch die Symptome selbst verbessern lassen.

Insgesamt spiegeln die Beiträge die Breite somatopsychologischer Fragestellungen und klinischer Anwendungsbereiche wider. Perspektiven für die psychotherapeutische Praxis sehen wir neben der weiteren Etablierung evidenzbasierter Ansätze im Bereich der stationären Versorgung v. a. in ihrer verstärkten Integration in die ambulante Versorgung.

\section{Korrespondenzadresse \\ Prof. Dr. Thomas Fydrich}

Institut für Psychologie, Humboldt-Universität zu Berlin

Rudower Chaussee 18, 12489 Berlin

fydrich@hu-berlin.de 


\section{Literatur}

Alexander F (1950) Psychosomatic medicine. Its principles and applications. Norton, New York

Birk L (1973) Biofeedback: behavioral medicine. Grune \& Stratton, New York

Bundespsychotherapeutenkammer (2006) Entwurf einer Weiterbildungsordnung. Unveröffentlichtes Manuskript

Ehlert U (2003) Verhaltensmedizin. Springer, Berlin Heidelberg New York Tokio

Engel GL (1977) The need for a new medical model: a challenge for biomedicine. Science 196: 129-136

Freud S, Breuer J (1895) Studien über Hysterie. Deuticke, Leipzig Wien

Groddeck (1923) Das Buch vom Es. Internationaler Psychoanalytischer Verlag, Leipzig Wien Zürich

Miltner W, Birbaumer N, Gerber WD (1986) Verhaltensmedizin. Springer, Berlin Heidelberg New York

Schwartz GE, Weiss SM (1978) Yale Conference on Behavioral Medicine. A proposed definition and statement of goals. J Behav Med 1: 3-12

Schwarzer R (2004) Psychologie des Gesundheitsverhaltens, 3. Aufl. Hogrefe, Göttingen

Uexkuell T von (Hrsg) (1979) Lehrbuch der psychosomatischen Medizin. Urban \& Schwarzenberg, München

\section{Ausschreibung Forschungspreis „MARIANNE-RINGLER-PREIS FÜR FORSCHUNG IN DER PSYCHOTHERAPIE 2010"}

Im Sinne seiner Zielsetzungen verleiht der Marianne Ringler Forschungsförderungsverein im Jahr 2010 einen Forschungspreis in der Höhe von 3.000,- Euro.

Der Marianne Ringler Forschungsförderungsverein setzt hiermit einen Forschungsförderungspreis aus für den - nach Maßgabe der Entscheidung des wissenschaftlichen Beirats - besten wissenschaftlichen Projektantrag auf dem Gebiet der Psychotherapie, insbesondere der Psychosomatik.

Der Forschungsförderungspreis für Projektanträge auf dem Gebiet der Psychotherapie, Psychoanalyse, Psychosomatik sowie Psychoonkologie wird insbesondere verliehen für empirische Forschungsprojekte aus der klinischen Praxis, Einzelfalldarstellungen, theoretische Abhandlungen und Literaturzusammenstellungen. Jede/r Verfasser/in kann nur einen Antrag einreichen.

Die eingereichten Projektanträge müssen in deutscher oder englischer Sprache verfasst sein und sollten einen Mindestumfang von 15 Seiten haben, sowie jedenfalls einen publikationsfähigen Abstract in deutscher und englischer Sprache umfassen.

Eingereicht werden dürfen Anträge für Projekte, die innerhalb von 24 Monaten fertig gestellt werden können. Ein detaillierter Zeitplan und Kostenplan ist beizulegen. Wird der Zeitplan nicht eingehalten, ist der Forschungspreis an den Verein zurückzuzahlen. Bereits fertig gestellte Projekte sind nicht antragsberechtigt.

Die Verwendung des Preisgeldes für das eingereichte Projekt ist durch entsprechende Originalbelege bei Projektabschluss nachzuweisen.

Die Genehmigung des Projekts durch die zuständige Ethikkommission ist dem Antrag beizulegen.

Der/die Verfasser/in sichert mit der Einreichung dem Marianne Ringler Forschungsförderungsverein zu, die publikationsreife Arbeit oder Auszüge daraus in der Homepage http://www.marianneringlerpreis.eu sowie http://www.sfu.ac.at oder in Papierform zu veröffentlichen. Entsprechende Texte sind dem Verein nach Abschluss des Projekts innerhalb von 24 Monaten nach Preisverleihung zur Verfügung zu stellen.

Die vom wissenschaftlichen Beirat des Marianne Ringler Forschungsförderungsvereins ausgewählte Arbeit wird mit einem Betrag von 3.000,- EUR ausgezeichnet. Der Betrag kann geteilt werden, wenn dem wissenschaftlichen Beirat keine eindeutig bessere Arbeit vorliegt.

Einsendeschluss ist der 10. September 2010. Der wissenschaftliche Beirat trifft die Entscheidung innerhalb von vier Wochen. Der Preis wird am 11. November 2010 in der Sigmund Freud Privatuniversität verliehen. Die Anträge sind per e-mail inklusive Lebenslauf einzureichen unter: ringler@marianneringlerpreis.eu. 